\title{
EDITORIAL ANNOUNCEMENT \\ The STROBE Initiative \\ STrengthening the Reporting of OBservational studies in Epidemiology (STROBE)
}

Much medical research is observation. The reporting of observation studies is often of insufficient quality. Poor reporting hampers the assessment of the strengths and weaknesses of a study and the generalizability of its results. Taking into account empirical evidence and theoretical considerations, a group of methodologists, researchers and editors developed the STrengthening the Reporting of OBservational studies in Epidemiology (STROBE) recommendations to improve the quality of reporting observation studies.

The STROBE statement [1] consists of a checklist of 32 items which relate to the title, abstract, introduction, methods, results and discussion sections of articles. Eighteen items are common to cohort studies, case-control studies and cross-sectional studies and four are specific to each of the three study designs. The STROBE statement provides guidance to authors about how to improve the reporting of observational studies and facilitates critical appraisal and interpretations of studies by reviewers, journal editors and readers.

An explanatory and elaboration document [2] is intended to enhance the use, understanding and dissemination of the STROBE statement. The meaning and rationale for each checklist item are presented. For each item, one or several published examples and, where possible, references to relevant empirical studies and methodological literature are provided. Examples of useful flow diagrams are also included. The STROBE statement, the explanatory document and the associated website should be helpful resources to improve reporting of observational research.

Epidemiology \& Infection is pleased to subscribe to this initiative. All authors are asked to ensure that papers submitted conform to these useful guidelines. Reviewers will be recommended to refer to them when assessing papers submitted to Epidemiology \& Infection.

NORMAN NOAH

Senior Editor

\section{References}

1. Strobe statement and website (www.strobe-statement. org).

2. Explanatory document (http://www.bmj.com/cgi/ content/full/335/7624/806). 